\title{
How to evaluate physical fitness without a stress test?
}

\author{
Judith B. Fischer $\cdot$ Bernhard Friedli • \\ Niklas F. Ehl $\cdot$ Michael J. Zellweger
}

Received: 3 September 2010/Accepted: 29 November 2010/Published online: 9 December 2010

(C) Springer Science+Business Media, B.V. 2010

\begin{abstract}
To assess cardiorespiratory fitness (CF), usually a stress test is necessary. Our aims were to assess $\mathrm{CF}$ in a patient population with suspected or known coronary artery disease (CAD) based on a questionnaire (quest); to compare estimated $\mathrm{CF}$ with achieved workloads, and to evaluate its prediction of stress modality (physical/pharmacologic). Consecutive 612 patients undergoing myocardial perfusion SPECT (MPS) completed quest. They first chose one category which best described their daily physical activities. The second part contained patient characteristics (gender, age, BMI, and resting heart rate). An activity score was calculated and metabolic equivalents (METs) were estimated. Estimated and achieved results were compared. Patients with pharmacologic test $(\mathrm{n}=208)$ provided a lower estimate of their performance than physically stressed patients $(\mathrm{n}=$ 404): $7.0 \pm 2.1$ and $8.2 \pm 2.3$ METs, respectively $(P<0.0001)$. The latter showed a good correlation between estimated and achieved METs $(\mathrm{r}=0.63$, $P<0.0001)$. Regarding prediction of the stress modality, area under the curve (ROC) was 0.65 $(P<0.0001)$. The quest can easily be applied in daily
\end{abstract}

There was no industry sponsoring of this study.

J. B. Fischer · B. Friedli · N. F. Ehl ·

M. J. Zellweger ( $\square)$

Cardiology Department, University Hospital Basel,

Petersgraben 4, 4031 Basel, Switzerland

e-mail: mzellweger@uhbs.ch practice to assess $\mathrm{CF}$ in a patient population with $\mathrm{CAD}$ and for estimating whether an adequate physical stress test can be carried out.

Keywords Cardiorespiratory fitness - Physical activity questionnaire - Coronary artery disease . Stress testing - Myocardial perfusion SPECT

$\begin{array}{ll}\text { Abbreviations } \\ \text { METs } & \text { Metabolic equivalents } \\ \text { MPS } & \begin{array}{l}\text { Myocardial perfusion SPECT } \\ \text { SPECT }\end{array} \\ & \begin{array}{l}\text { Single photon emission computed } \\ \text { tomography }\end{array} \\ \text { SSS } & \text { Summed stress score }\end{array}$

\section{Introduction}

Low cardiorespiratory fitness is a strong predictor of mortality [1-6]. Physically inactive individuals are more likely to suffer an early death from a cardiovascular or non-cardiovascular disease than those who are physically active [1, 4, 7-9]. The correlation between low cardiorespiratory fitness and increased mortality is as strong as correlations between mortality and regular risk factors, such as hypertension, diabetes, and obesity [1]. In addition, the higher the total energy expenditure, the lower the risk of coronary events $[1,3,8]$, but also of overall mortality $[2,3,10]$.

The evaluation of cardiorespiratory fitness is important in different aspects $[8,11]$. Indications may be the 
prognostic evaluation $[1,7,8,11]$, planning of a stress test [11] or preoperative risk stratification [12]. However, to assess cardiorespiratory fitness of a patient, usually a stress test is necessary [11], which may cause problems regarding feasibility or logistics [13]. Therefore it might be easier and also reliable if the physical activity is estimated with the patient's baseline characteristics and a questionnaire of the patient's daily physical activities [13].

A number of studies have been published using questionnaires to determine the cardiorespiratory fitness of patients [13-15]: e.g. questionnaires for randomly selected subjects $[13,16,17]$, for patient populations with cancer [18], and patients with congestive heart failure $[14,19,20]$. In contrast, few papers have been published using questionnaires to evaluate physical activity in patients with (suspected or) known coronary artery disease [15, 21].

The aims of the present study were as follows: (1) to use and test questionnaires that are easily conceived and quickly completed in daily practice in a patient population evaluated for coronary artery disease, (2) to compare estimated cardiorespiratory fitness with achieved workloads, (3) to evaluate if these questionnaires could be used to predict if a patient is able to undergo an adequate physical stress test or rather a pharmacologic one, and (4) to test and apply the questionnaires for preoperative evaluation.

\section{Methods}

Study population

All consecutive patients undergoing myocardial perfusion SPECT from January 30th until July 10th, 2008 at the University Hospital of Basel, Switzerland, were evaluated. They underwent coronary artery disease evaluation and therefore were referred for an MPS at the discretion of their physicians. 41 patients with complete left bundle branch block were excluded from the analysis because they had to undergo pharmacologic stress for protocol reasons.

\section{Exercise MPS protocol}

Rest SPECT was performed after administration of $111 \mathrm{MBq}$ of ${ }^{201} \mathrm{Tl}$ [22]. ${ }^{201} \mathrm{Tl}$ SPECT was performed $10 \mathrm{~min}$ after tracer injection [22]. A symptom-limited bicycle exercise test was performed, using routine protocols (with a stepwise increase of the workload). The monitoring included a 12-lead electrocardiogram each minute of exercise and continuous monitoring of the electrocardiogram throughout the test [22]. At nearmaximal exercise, a 740-MBq dose of ${ }^{99} \mathrm{~m}$ Tc-sestamibi was injected, and exercise was continued for at least an additional minute after injection [22]. ${ }^{99 \mathrm{~m}} \mathrm{Tc}$-sestamibi SPECT imaging was begun 15-30 min later [9].

Regarding cardiorespiratory fitness, METs achieved were calculated using the formula $\mathrm{VO}_{2} / 3.5$ - body weight $(\mathrm{kg})\left[\mathrm{VO}_{2}=5.8 \cdot\right.$ body weight $(\mathrm{kg})+151+10.5$. watts achieved].

\section{Pharmacologic MPS protocol}

Patients were informed not to consume any products containing caffeine $24 \mathrm{~h}$ before testing [22]. After rest-imaging, adenosine was infused $(140 \mu \mathrm{g} / \mathrm{kg} / \mathrm{min}$ for $6 \mathrm{~min}$ ), and ${ }^{99 \mathrm{~m}} \mathrm{Tc}$-sestamibi was injected at the end of the third minute of infusion [22]. 60 min later, patients underwent SPECT imaging [9, 23]. If possible the adenosine stress was combined with low level physical exercise (in general 25 watts).

Whenever possible, patients were instructed to pause $\beta$-blocking agents and calcium-antagonists $48 \mathrm{~h}$ and nitrates $24 \mathrm{~h}$ before the beginning of the test, regardless of stress modality [22]. At rest, at the end of each exercise stage and at maximal exercise, blood pressure was registered, and so was the electrocardiogram, according to current guidelines [12, 24]. Peak ST-segment change at $80 \mathrm{~ms}$ after the $\mathrm{J}$ point was stated as horizontal, up or downsloping [9, 23].

\section{MPS evaluation}

SPECT was conducted with a circular $180^{\circ}$ acquisition, as previously described [23]. Two energy windows for Tl-201 were used during imaging, containing a $30 \%$ window centred on the $70-\mathrm{keV}$ peak and a $20 \%$ window centred on the $167-\mathrm{keV}$ peak [22]. A $15 \%$ window centred on the $140-\mathrm{keV}$ peak was utilised for ${ }^{99 \mathrm{~m}}$ Tc-sestamibi SPECT [22]. Perfusion images were scored using a 17-segment model with a 5-point scale $(0=$ normal, $1=$ mildly reduced tracer uptake, $2=$ moderately reduced uptake, $3=$ severely reduced uptake, $4=$ no uptake) [22]. Each segment represents $5.9 \%$ of the left ventricle [23]. By adding the scores of the 17 segments in the stress and rest images, the 
overall summed stress score (SSS) was calculated [22]. SSS $<4$ was considered normal, SSS 4-8 mildly abnormal, 9-13 moderately abnormal and SSS $>13$ was considered severely abnormal [25]. Post-stress LVEF, EDV and ESV were automatically calculated by QGS $^{\mathrm{TM}}[26,27]$.

Development of the physical activity scale of questionnaire 1

In this paper, we refer to the physical activity scale of this questionnaire as "questionnaire 1". It was developed by selecting frequent daily life activities, categorized into exertion levels by 2543 randomly selected people [16]. The physical activity scale was divided into 9 levels, ranging from 0.9 METs to $>6$ METs (Table 1). The composition of the physical activity scale allowed the patient to fill in the hours and minutes on each level on an average $24 \mathrm{~h}$ weekday. Validation occurred by interviewing 10 volunteers and further on by recruiting 39 volunteers to establish correlation between MET-time estimated and calculated, which was high [16].

While evaluating the questionnaires for our study, we asked the authors of the physical activity scale for a German translation of the originally Danish questionnaire, which we kindly received. By estimating METs on the highest level on the physical activity scale,

Table 1 Questionnaire 1

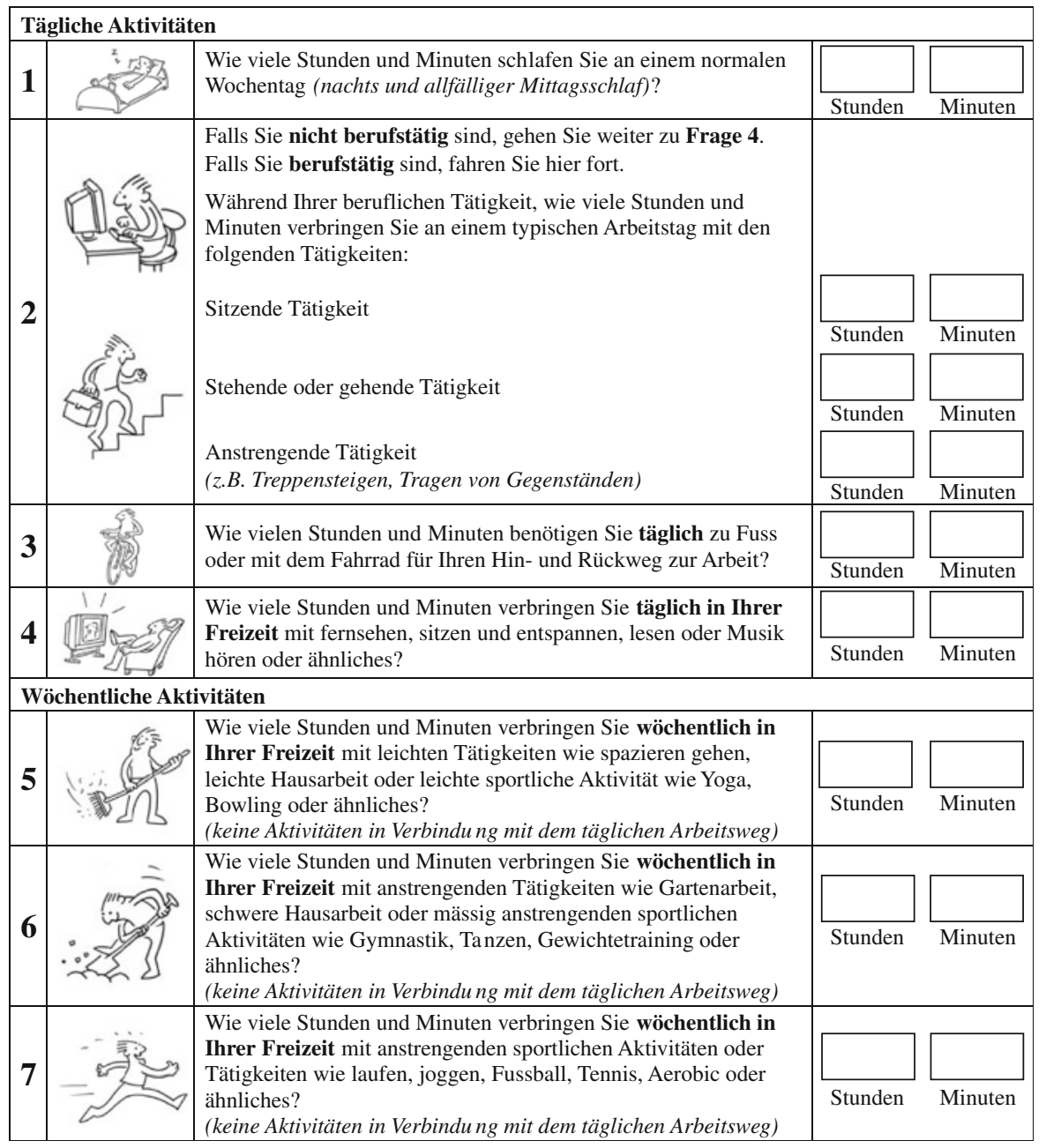

Modified from Aadahl et al. [16] 
which was defined as $>6$ METs, we utilized 8.0 METs for calculation. With questionnaire 1 , finally an activity scale was calculated. Oftentimes the hours filled in by the patients did not add up to $24 \mathrm{~h}$. We approached this problem by multiplying the surplus or missing time with 2.0 MET and adding it to or subtracting it from the excessive or missing hours and minutes [16].

Development of physical activity score of questionnaire 2

In this paper, we refer to the fitness evaluation as "questionnaire 2". When developing this non-exercise test model, data from three previous studies were used [13], consisting of a total of 49,759 volunteers (1863 NASA participants, 46,190 subjects from the Aerobics Center Longitudinal Study, and 1706 participants from the Allied Dunbar National Fitness Survey) [28-31]. They all underwent treadmill testing and provided data about gender, age, height, weight, resting heart rate and self-reported physical activity levels [13, 28-31].

The non-exercise test model consists of two parts. The first part contains a physical activity score where people have to choose one category which best describes the usual pattern of daily physical activities. The second part contains historical data, including patient characteristics (gender, age, BMI, resting heart rate) (Table 2). Based on these two parts, estimated METs were calculated [13]. If not otherwise mentioned we refer to the two parts of the fitness evaluation when mentioning "questionnaire 2". Since there was no German version of the originally English questionnaire available, we therefore translated it ourselves.

\section{Results}

Baseline characteristics

A total of 845 consecutive patients was evaluated after having obtained informed consent. Of these, 653 (77\%) patients completed the questionnaires correctly. Forty-one patients completed the questionnaires correctly but were not included into the analysis due to a left bundle branch block. Of the remaining 192 patients who were not included, 49 did not complete the questionnaire because of physical inability (e.g. no glasses at the hospital) 132 patients because of language barriers, and 11 subjects declined.

The patient baseline characteristics are summarized in Table 3, comparing patients undergoing physical $(66 \%)$ and pharmacologic (34\%) stress testing.

Patients with a pharmacologic stress test $(n=208)$ were significantly older, more often suffered from shortness of breath and had a higher cardiovascular risk profile than patients who underwent a bicycle stress test $(n=404)$. The former also more often were under therapy with oral anticoagulation, ß-blocking agents, nitrates, and diuretics.
Table 2 Questionnaire 2

Shortened from original (Jurca et al.) [13]

\begin{tabular}{|c|c|}
\hline \multicolumn{2}{|c|}{ Step 1: Physical activity score } \\
\hline Level 1 & Inactive \\
\hline Level 2 & $\begin{array}{l}\text { Low level of exertion, } \\
\leq 10 \mathrm{~min} \text { at a time }\end{array}$ \\
\hline Level 3 & $\begin{array}{l}\text { Aerobic exercises } \\
20-60 \mathrm{~min} / \text { week }\end{array}$ \\
\hline Level 4 & $\begin{array}{l}\text { Aerobic exercises } \\
1-3 \mathrm{~h} / \text { week }\end{array}$ \\
\hline Level 5 & $\begin{array}{l}\text { Aerobic exercises } \\
>3 \mathrm{~h} / \text { week }\end{array}$ \\
\hline \multicolumn{2}{|c|}{$\begin{array}{l}\text { In addition to the above calculated scores, } \\
\text { the following variables are added in a weighted } \\
\text { manner resulting in an estimate of METs: }\end{array}$} \\
\hline \multicolumn{2}{|c|}{ Step 2: Estimated MET level of cardiorespiratory fitness (from step 1) } \\
\hline \multicolumn{2}{|l|}{ Gender } \\
\hline \multicolumn{2}{|l|}{ Age } \\
\hline \multicolumn{2}{|l|}{ Body mass index } \\
\hline Resting heart rate & \\
\hline
\end{tabular}


Table 3 Baseline characteristics
$B M I$ body mass index, $C A D$ coronary artery disease, $M I$ myocardial infarction, $\mathrm{Ca}$ Calcium, $A C E$ angiotensin converting enzyme, $A T 2$ Angiotensin 2

\begin{tabular}{|c|c|c|c|c|}
\hline Variables & $\begin{array}{l}\text { Total } \\
(\mathrm{n}=612)\end{array}$ & $\begin{array}{l}\text { Bicycle } \\
(\mathrm{n}=404)\end{array}$ & $\begin{array}{l}\text { Pharmacologic } \\
(\mathrm{n}=208)\end{array}$ & $P$ \\
\hline Sex (male) & $66 \%$ & $67 \%$ & $64 \%$ & 0.53 \\
\hline Age (years) & $65 \pm 10$ & $63 \pm 10$ & $69 \pm 9$ & $<0.0001$ \\
\hline BMI $\left(\mathrm{kg} / \mathrm{m}^{2}\right)$ & $28 \pm 5$ & $28 \pm 5$ & $28 \pm 5$ & 0.07 \\
\hline Typical angina & $19 \%$ & $17 \%$ & $22 \%$ & 0.19 \\
\hline Atypical angina & $28 \%$ & $30 \%$ & $25 \%$ & 0.18 \\
\hline Shortness of breath & $57 \%$ & $51 \%$ & $71 \%$ & $<0.0001$ \\
\hline Known CAD & $41 \%$ & $40 \%$ & $45 \%$ & 0.26 \\
\hline Prior revascularisation & $37 \%$ & $37 \%$ & $37 \%$ & 0.93 \\
\hline Prior MI & $25 \%$ & $24 \%$ & $27 \%$ & 0.43 \\
\hline Diabetes mellitus & $25 \%$ & $22 \%$ & $29 \%$ & 0.092 \\
\hline Hypercholesterolemia & $62 \%$ & $62 \%$ & $63 \%$ & 1.0 \\
\hline Hypertension & $72 \%$ & $69 \%$ & $80 \%$ & 0.004 \\
\hline Positive family history & $33 \%$ & $37 \%$ & $25 \%$ & 0.005 \\
\hline Smoking & $18 \%$ & $17 \%$ & $20 \%$ & 0.37 \\
\hline Anticoagulation & $13 \%$ & $9 \%$ & $20 \%$ & $<0.0001$ \\
\hline Aspirin & $70 \%$ & $71 \%$ & $68 \%$ & 0.52 \\
\hline ß-blocking agents & $61 \%$ & $53 \%$ & $76 \%$ & $<0.0001$ \\
\hline Nitrates & $14 \%$ & $11 \%$ & $20 \%$ & 0.002 \\
\hline Ca-Antagonists & $21 \%$ & $19 \%$ & $25 \%$ & 0.092 \\
\hline ACE inhibitors & $31 \%$ & $29 \%$ & $34 \%$ & 0.20 \\
\hline AT2-inhibitors & $26 \%$ & $24 \%$ & $30 \%$ & 0.10 \\
\hline Statins & $59 \%$ & $57 \%$ & $63 \%$ & 0.23 \\
\hline Diuretics & $39 \%$ & $33 \%$ & $50 \%$ & $<0.0001$ \\
\hline
\end{tabular}

Estimates of cardiorespiratory fitness

In Fig. 1 the estimates of physical performance are summarized.

In patients who were able to undergo a bicycle stress test $(\mathrm{n}=404)$, METs estimated and METs achieved were compared. In Fig. 2 the missing correlation of the physical activity scale of questionnaire 1 and the achieved METs is shown $(\mathrm{r}=0.06$, $P=0.23$ ). In contrast, there was a good correlation between estimated METs of questionnaire 2 and the achieved METs ( $\mathrm{r}=0.63, P<0.0001$; Fig. 3 ).

A Bland-Altman plot of the estimated METs and the difference of estimated and achieved METs of questionnaire 2 is depicted in Fig. 4. In patients with an estimated workload $\leq 8$ METs, the estimate was rather underestimated. In patients with an estimated workload $>8$ METs, the estimate was rather overestimated. 
Table 4 Stresstest variables

$R B B B$ right bundle branch block, METs metabolic equivalents, SSS summed stress score, SRS summed rest score, $S D S$ summed difference score, $E F$ ejection fraction, $E D V$ enddiastolic volume, $E S V$ endsystolic volume

\begin{tabular}{|c|c|c|c|c|}
\hline & $\begin{array}{l}\text { Total } \\
(\mathrm{n}=612)\end{array}$ & $\begin{array}{l}\text { Bicycle } \\
(\mathrm{n}=404)\end{array}$ & $\begin{array}{l}\text { Pharmacologic } \\
(n=208)\end{array}$ & $P$ \\
\hline Sinus Rhythm & $91 \%$ & $93 \%$ & $85 \%$ & $<0.0001$ \\
\hline Q-wave & $17 \%$ & $17 \%$ & $17 \%$ & 1.0 \\
\hline Right bundle branch block & $4 \%$ & $5 \%$ & $3 \%$ & 0.53 \\
\hline Angina during testing & $12 \%$ & $9 \%$ & $19 \%$ & $<0.0001$ \\
\hline Watts reached & & $142 \pm 48$ & & \\
\hline METs achieved & & $7.6 \pm 1.7$ & & \\
\hline Basic heartrate (bpm) & $77 \pm 15$ & $78 \pm 14$ & $75 \pm 18$ & 0.07 \\
\hline Max. heartrate (bpm) & & $143 \pm 16$ & & \\
\hline $\begin{array}{l}\text { Basic systolic bloodpressure } \\
(\mathrm{mmHg})\end{array}$ & $125 \pm 22$ & $125 \pm 22$ & $125 \pm 22$ & 0.97 \\
\hline $\begin{array}{l}\text { Max. systolic bloodpressure } \\
\quad(\mathrm{mmHg})\end{array}$ & & $196 \pm 32$ & & \\
\hline Significant ST-changes & $19 \%$ & $19 \%$ & $23 \%$ & 0.23 \\
\hline SSS (median) & 0 & 0 & 3.00 & $<0.0001$ \\
\hline SRS (median) & 0 & 0 & 0 & 0.009 \\
\hline SDS (median) & 0 & 0 & 0 & 0.002 \\
\hline $\mathrm{EF}(\%)$ & $58 \pm 11$ & $58 \pm 11$ & $56 \pm 12$ & 0.07 \\
\hline EDV & $97 \pm 38$ & $95 \pm 37$ & $102 \pm 42$ & 0.05 \\
\hline ESV & $45 \pm 29$ & $43 \pm 27$ & $48 \pm 32$ & 0.037 \\
\hline
\end{tabular}

Table 5 Questionnaires

METs metabolic equivalents

\begin{tabular}{lllll}
\hline Variables & $\begin{array}{l}\text { Total } \\
(\mathrm{n}=612)\end{array}$ & $\begin{array}{l}\text { Bicycle } \\
(\mathrm{n}=404)\end{array}$ & $\begin{array}{l}\text { Pharmacologic } \\
(\mathrm{n}=208)\end{array}$ & $P$ \\
\hline $\begin{array}{l}\text { Questionnaire 1 } \\
\begin{array}{l}\text { Physical activity scale } \\
\text { (median) }\end{array}\end{array}$ & 43 & 43 & 41 & $<0.0001$ \\
$\begin{array}{l}\text { Questionnaire 2 } \\
\text { Activity score (part 1) }\end{array}$ & $1.4 \pm 1.1$ & $1.6 \pm 1.1$ & $1.2 \pm 1.0$ & $<0.0001$ \\
Estimated METs (part 2) & $7.8 \pm 2.3$ & $8.2 \pm 2.3$ & $7.0 \pm 2.1$ & $<0.0001$ \\
\hline
\end{tabular}

In Fig. 5 the comparison of estimated and achieved METs in relation to the extent of coronary artery disease is shown. Interestingly, no difference between the estimated and achieved METs was evident when the different SSS categories were compared. However, within the four SSS categories the estimated and reached METs were significantly different, although the absolute difference was small.

\section{Prediction of stress modality}

In Fig. 6 the predictive accuracies of questionnaire 1 and questionnaire 2 regarding the stress modality used are shown. By receiver operating characteristics
(ROC), the area under the curve (AUC) was 0.60 and 0.65 , respectively, (both $P<0.0001$ ).

Since questionnaire 2 consists of the patients' physical activity score and historical patient data, predictive power of only basic variables was tested in a first step. An AUC of 0.61 was obtained. Adding the historical information increased the AUC to 0.65 (Fig. 7).

\section{Preoperative evaluation}

In a small percentage of the patient population ( $\mathrm{n}=38 ; 6.3 \%$ ), the estimated physical activity did not reach 4.0 METs, which is an important threshold 


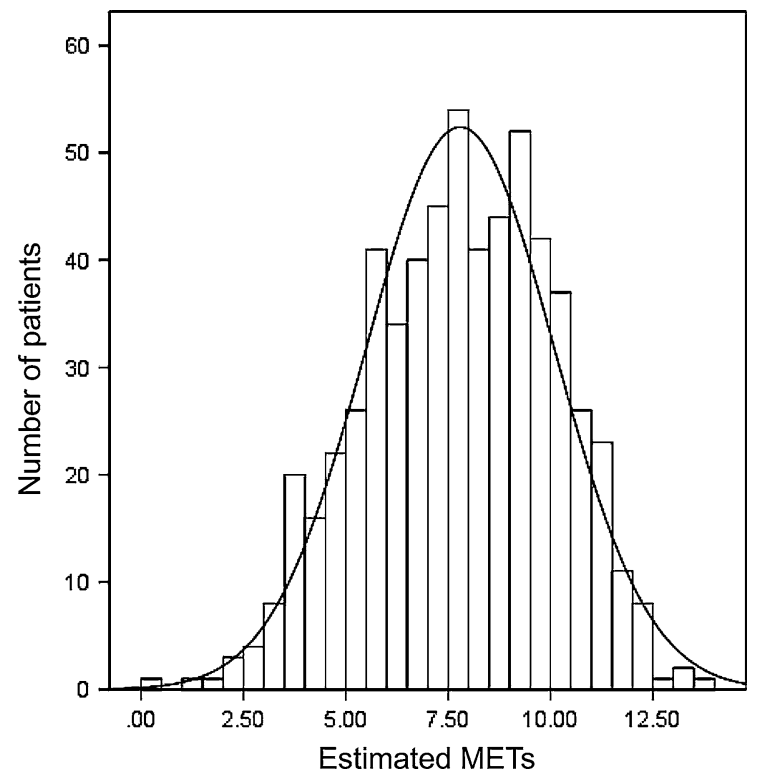

Fig. 1 Distribution of estimated METs in all patients, $n=612$

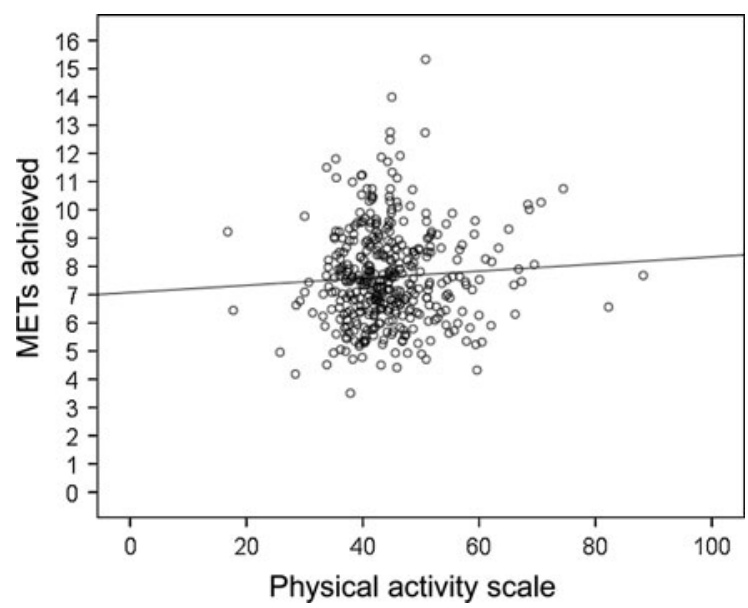

Fig. 2 Correlation of the physical activity scale and achieved METs as assessed by questionnaire $1, \mathrm{n}=404 . \mathrm{r}=0.06$, $\mathrm{r}^{2}=0.004, P=0.23$

for preoperative risk stratification [32], as shown in Fig. 1. Of those patients with an estimate $<4$ METs, $70.6 \%$ achieved $\geq 4$ METs during exercise testing. Of those patients with an estimate $\geq 4$ METs, $11.4 \%$ achieved $<4$ METs during exercise testing.

The subgroups are too small to further evaluate this patient population. Therefore, no variables could be evaluated as independent predictors of the wrong estimate of the workload.

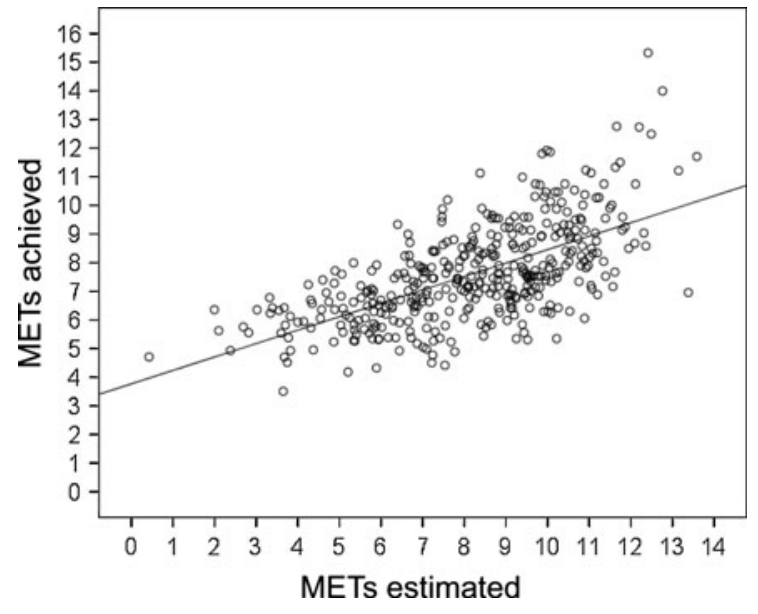

Fig. 3 Correlation of METs estimated/achieved by questionnaire $2, \mathrm{n}=404 . \mathrm{r}=0.63, \mathrm{r}^{2}=0.4, P<0.0001$

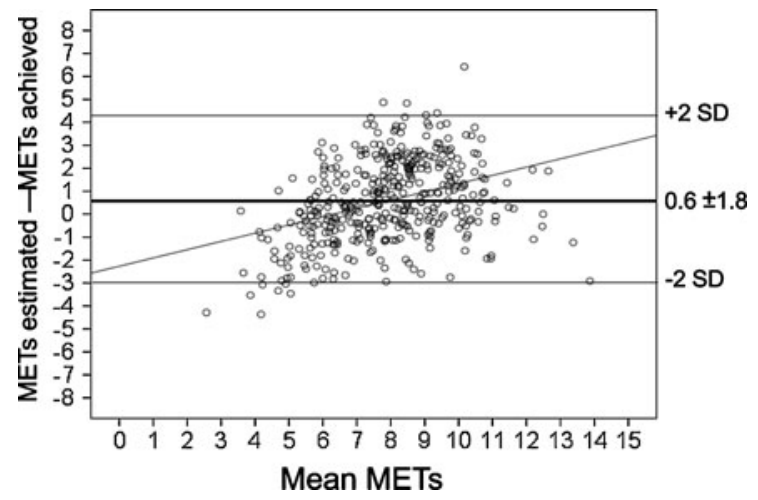

Fig. 4 Bland-Altman plot of mean METs (estimated and achieved) and the difference of METs estimated and METs achieved (questionnaire 2), $\mathrm{n}=404$

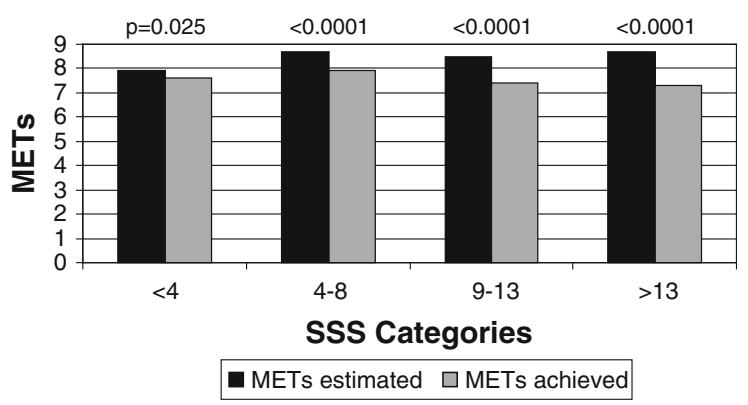

Fig. 5 Comparison of METs estimated and METs achieved regarding extent of coronary artery disease as assessed by SSS (summed stress score) category $(n=404)$ 


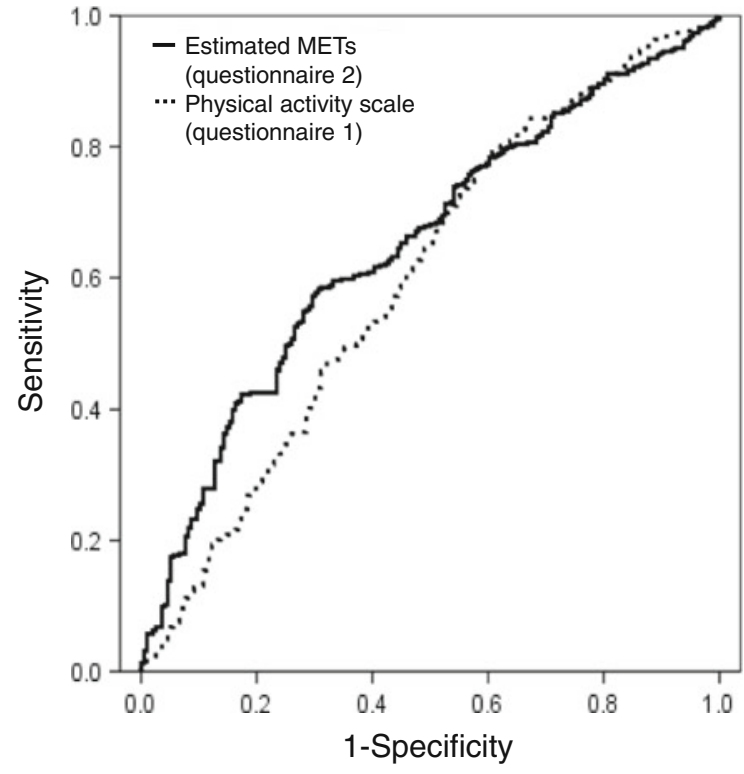

Fig. 6 ROC (receiver operating characteristics) curve of accuracy of questionnaire 1 and 2 regarding stress modality used. AUC (area under the curve) of questionnaire $2=0.65$, $P<0.0001$; questionnaire $1=0.60, P<0.0001$

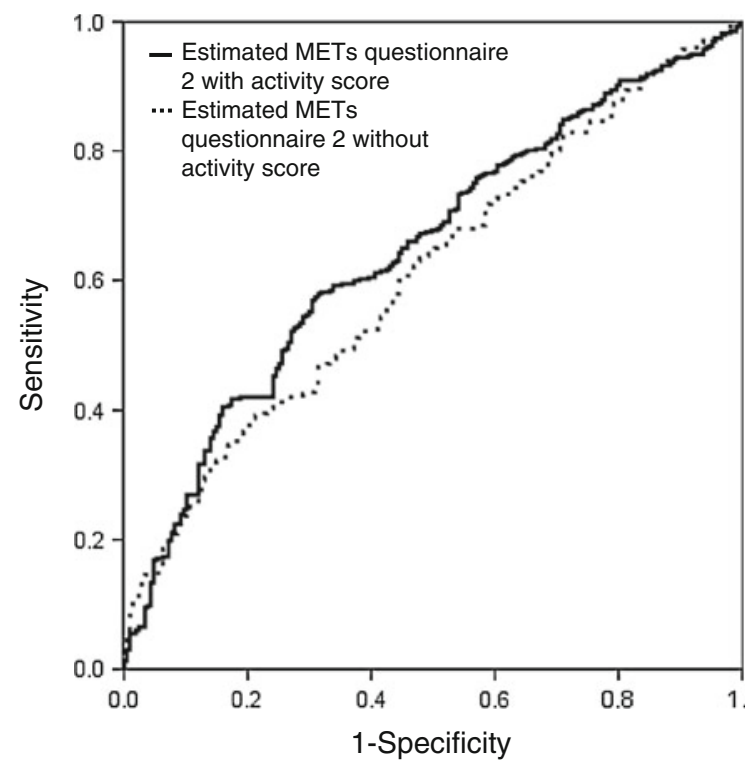

Fig. 7 ROC curve of questionnaire 2 with and without step 1 . Step 1 contains a physical activity score, step 2 consists of historical patient data. AUC without step $1=0.61$; AUC with step $1=0.65$

\section{Discussion}

The correlation between low cardiorespiratory fitness and high mortality has been demonstrated by a number of studies $[1,4-8,10]$. It was shown that the patients, who failed to achieve 6 METs or more during exercise treadmill testing, had a significantly higher percentage both for all-cause and cardiac death than patients who are physically fit [10]. In addition, the worse patients performed on the treadmill, the more increased the risk of mortality [10]. Generally a stress test is needed to evaluate cardiorespiratory fitness. The present study demonstrates that questionnaires evaluating fitness may be used as a surrogate for stress testing regarding evaluation of cardiorespiratory fitness. To our knowledge it is the first study that compared estimated fitness with the actual performance in patients with suspected or known coronary artery disease.

Questionnaire 1 allows a certain evaluation of the patient's cardiorespiratory fitness, especially to answer the question whether or not the patient will be able to undergo a sufficient physical stress test. However, it is not suitable to predict the patient's performance. In contrast, questionnaire 2 can well be used to estimate METs and to predict the type of stress test.

The patients usually required 5-10 min to fill in the two questionnaires. Both questionnaires were correctly completed in the main part of the patient population, with few patients having minor difficulties. The main problem was caused by language barrier, whereas questionnaires translated into different languages would provide a solution. Another hurdle was impaired vision, which usually would not have been a problem if the patients had always had their glasses with them. In general, the questionnaires can readily be integrated into daily practice and cause very little additional expenses. Legibility, language and accordance of the questionnaires to ethnicity have to be considered.

When estimating workloads for prognostic purposes, questionnaire 2 seems to be more reliable than questionnaire 1, since estimated METs are more accurate in the former. Meanwhile, patients with an estimated workload $\leq 8$ tended to be underestimated, whereas patients with an estimated workload $>8$ were more likely to be overestimated.

Not many questionnaires that evaluate cardiorespiratory fitness and which are concise and easily 
understood are available. In our opinion, we selected the ones that were the simplest and best validated in non-CAD populations. When questionnaire 2 was published, it was discussed in that paper that additional work would be needed to evaluate it in daily practice; to establish this non-exercise test model as a predictor for physical activity [13]. Originally, validation occurred both in questionnaire 1 as well as in questionnaire 2 with young and healthy people who in general have a good cardiorespiratory fitness [13, 16]. The patients in our study were significantly older, less fit and suffered from suspected or known coronary artery disease (original questionnaire 1 population: mean age $=40$ years, mean total $24 \mathrm{~h}$ MET-time $=50$, which is consistent with relatively fit persons [16]; in contrast, original questionnaire 2 population: mean age $=43$ years, mean achieved METs $=11$ [13]; our study population: mean age $=65$ years, mean achieved METs $=8$ ). When taking this into consideration, it is remarkable, how accurate the prediction of the present patients' cardiorespiratory fitness with these questionnaires is. People with a higher level of physical fitness have a lower risk to die of coronary artery events [21]. Overall, questionnaire 2 allows a good estimation of the patient's physical activity and may therefore be considered as a possible risk stratification tool.

Both questionnaires can be applied for estimating whether an adequate bicycle stress test can be carried out or if the patient has to undergo a pharmacologic stress test.

Regarding questionnaire 2, the non-exercise test model consists of the patients' physical activity score and historical patient data [13]. The combination of them is more precise in prediction than just memorable facts. Nevertheless, the additional element of the memorable data increased the accuracy of prediction significantly.

The questionnaires can be used to decide with which stress modality a patient should be evaluated. The ROC curves demonstrated better results for questionnaire 2 than for questionnaire 1. A limiting factor for the prediction of the stress modality is that the adequacy of physical stress test is not only based on the workload but also on the age adapted threshold of the heart rate (in general $85 \%$ of the maximal heart rate) that needs to be achieved. This may in part explain that the estimated workload of physically and pharmacologically stressed patients is only slightly different (e.g. a patient who performs well on the bicycle but does not reach the age adapted heart rate is switched to pharmacologic stress testing).

In a few cases, questionnaire 2 may be beneficial for preoperative risk stratification purposes. According to the guidelines, patients generally can undergo surgery without further testing if they accomplish 4 METs [32]. In daily practice, it is easiest to ask the patients if they can climb two flights of stairs. However, in certain cases questionnaire 2 might be useful to distinguish performance more clearly, as shown in Fig. 1.

As stated above, patients with an estimated workload $\leq 8$ tended to be underestimated. According to that finding, the majority of patients who estimated their physical performance to be $<4$ METs was wrong. Therefore, in these patients the questionnaire could not contribute to the decision making process in the preoperative setting. At the same time, only $11.4 \%$ of the patients with an estimation $\geq 4$ METs did not reach 4 METs during exercise testing, allowing the conclusion that if the patients estimate their performance to be $\geq 4$ METs, the majority of patients (almost 90\%) are able to achieve that threshold in reality and therefore can undergo surgery without further testing.

\section{Conclusion}

The questionnaires can easily be applied in daily practice to assess cardiorespiratory fitness in a patient population with suspected or known coronary artery disease and can also be used for estimating whether an adequate stress test can be carried out or if the patient has to undergo a pharmacologic stress test. Additionally, questionnaire 2 allows an accurate estimate of the patient's physical fitness and may therefore be considered as a possible risk stratification tool.

Conflict of interest None.

\section{References}

1. Laukkanen JA, Lakka TA, Rauramaa R, Kuhanen R, Venalainen JM, Salonen R, Salonen JT (2001) Cardiovascular fitness as a predictor of mortality in men. Arch Intern Med 161:825-831 
2. Lee IM, Paffenbarger RS Jr (2000) Associations of light, moderate, and vigorous intensity physical activity with longevity. The harvard alumni health study. Am J Epidemiol 151:293-299

3. Kokkinos P, Myers J, Kokkinos JP, Pittaras A, Narayan P, Manolis A, Karasik P, Greenberg M, Papademetriou V, Singh S (2008) Exercise capacity and mortality in black and white men. Circulation 117:614-622

4. Laukkanen JA, Rauramaa R, Salonen JT, Kurl S (2007) The predictive value of cardiorespiratory fitness combined with coronary risk evaluation and the risk of cardiovascular and all-cause death. J Intern Med 262:263-272

5. Nocon M, Hiemann T, Muller-Riemenschneider F, Thalau F, Roll S, Willich SN (2008) Association of physical activity with all-cause and cardiovascular mortality: a systematic review and meta-analysis. Eur J Cardiovasc Prev Rehabil 15:239-246

6. Sofi F, Capalbo A, Cesari F, Abbate R, Gensini GF (2008) Physical activity during leisure time and primary prevention of coronary heart disease: an updated meta-analysis of cohort studies. Eur J Cardiovasc Prev Rehabil 15:247-257

7. Laukkanen JA, Kurl S, Salonen R, Rauramaa R, Salonen JT (2004) The predictive value of cardiorespiratory fitness for cardiovascular events in men with various risk profiles: a prospective population-based cohort study. Eur Heart J 25:1428-1437

8. Lee IM, Sesso HD, Paffenbarger RS Jr (2000) Physical activity and coronary heart disease risk in men: does the duration of exercise episodes predict risk? Circulation 102:981-986

9. Zellweger MJ, Hachamovitch R, Kang X, Hayes SW, Friedman JD, Germano G, Pfisterer ME, Berman DS (2004) Prognostic relevance of symptoms versus objective evidence of coronary artery disease in diabetic patients. Eur Heart J 25:543-550

10. Snader CE, Marwick TH, Pashkow FJ, Harvey SA, Thomas JD, Lauer MS (1997) Importance of estimated functional capacity as a predictor of all-cause mortality among patients referred for exercise thallium single-photon emission computed tomography: Report of 3,400 patients from a single center. J Am Coll Cardiol 30:641-648

11. Fletcher GF, Balady GJ, Amsterdam EA, Chaitman B, Eckel R, Fleg J, Froelicher VF, Leon AS, Pina IL, Rodney R, Simons-Morton DA, Williams MA, Bazzarre T (2001) Exercise standards for testing and training: a statement for healthcare professionals from the American heart association. Circulation 104:1694-1740

12. Gibbons RJ, Balady GJ, Beasley JW, Bricker JT, Duvernoy WF, Froelicher VF, Mark DB, Marwick TH, McCallister BD, Thompson PD, Winters WL Jr, Yanowitz FG, Ritchie JL, Cheitlin MD, Eagle KA, Gardner TJ, Garson A Jr, Lewis RP, O'Rourke RA, Ryan TJ (1997) Acc/aha guidelines for exercise testing: executive summary. A report of the American college of cardiology/American heart association task force on practice guidelines (committee on exercise testing). Circulation 96:345-354

13. Jurca R, Jackson AS, LaMonte MJ, Morrow JR Jr, Blair SN, Wareham NJ, Haskell WL, van Mechelen W, Church TS, Jakicic JM, Laukkanen R (2005) Assessing cardiorespiratory fitness without performing exercise testing. Am J Prev Med 29:185-193
14. Green CP, Porter CB, Bresnahan DR, Spertus JA (2000) Development and evaluation of the kansas city cardiomyopathy questionnaire: a new health status measure for heart failure. J Am Coll Cardiol 35:1245-1255

15. Lip GY, Cader MZ, Lee F, Munir SM, Beevers DG (1996) Ethnic differences in pre-admission levels of physical activity in patients admitted with myocardial infarction. Int J Cardiol 56:169-175

16. Aadahl M, Jorgensen T (2003) Validation of a new selfreport instrument for measuring physical activity. Med Sci Sports Exerc 35:1196-1202

17. Mader U, Martin BW, Schutz Y, Marti B (2006) Validity of four short physical activity questionnaires in middleaged persons. Med Sci Sports Exerc 38:1255-1266

18. Elmqvist MA, Jordhoy MS, Bjordal K, Kaasa S, Jannert M (2009) Health-related quality of life during the last three months of life in patients with advanced cancer. Support Care Cancer 17:191-198

19. Rustoen T, Stubhaug A, Eidsmo I, Westheim A, Paul SM, Miaskowski C (2008) Pain and quality of life in hospitalized patients with heart failure. J Pain Symptom Manage 36:497-504

20. Schiffer AA, Pedersen SS, Widdershoven JW, Denollet J (2008) Type d personality and depressive symptoms are independent predictors of impaired health status in chronic heart failure. Eur J Heart Fail 10:802-810

21. Pitsavos C, Kavouras SA, Panagiotakos DB, Arapi S, Anastasiou CA, Zombolos S, Stravopodis P, Mantas Y, Kogias Y, Antonoulas A, Stefanadis C (2008) Physical activity status and acute coronary syndromes survival the greecs (greek study of acute coronary syndromes) study. J Am Coll Cardiol 51:2034-2039

22. Zellweger MJ, Kaiser C, Brunner-La Rocca HP, Buser PT, Osswald S, Weiss P, Mueller-Brand J, Pfisterer ME (2008) Value and limitations of target-vessel ischemia in predicting late clinical events after drug-eluting stent implantation. J Nucl Med 49:550-556

23. Zellweger MJ, Tabacek G, Zutter AW, Weinbacher M, Cron TA, Muller-Brand J, Pfisterer ME (2004) Evidence for left ventricular remodeling after percutaneous coronary intervention: effect of percutaneous coronary intervention on left ventricular ejection fraction and volumes. Int $\mathbf{J}$ Cardiol 96:197-201

24. Gibbons RJ, Balady GJ, Bricker JT, Chaitman BR, Fletcher GF, Froelicher VF, Mark DB, McCallister BD, Mooss AN, O'Reilly MG, Winters WL, Gibbons RJ, Antman EM, Alpert JS, Faxon DP, Fuster V, Gregoratos G, Hiratzka LF, Jacobs AK, Russell RO, Smith SC (2002) Acc/aha 2002 guideline update for exercise testing: summary article. A report of the American college of cardiology/American heart association task force on practice guidelines (committee to update the 1997 exercise testing guidelines). J Am Coll Cardiol 40:1531-1540

25. Hachamovitch R, Berman DS, Kiat H, Cohen I, Cabico JA, Friedman J, Diamond GA (1996) Exercise myocardial perfusion spect in patients without known coronary artery disease: incremental prognostic value and use in risk stratification. Circulation 93:905-914

26. Berman DS, Kiat H, Friedman JD, Wang FP, van Train K, Matzer L, Maddahi J, Germano G (1993) Separate acquisition rest thallium-201/stress technetium-99m sestamibi 
dual-isotope myocardial perfusion single-photon emission computed tomography: a clinical validation study. J Am Coll Cardiol 22:1455-1464

27. Kaufmann BA, Pfisterer ME, Viswanathan S, MullerBrand J, Zellweger MJ (2006) Stunning and left ventricular function-how long is the ventricle knocked out? Left ventricular function correlated with ischemic burden and recovery time after stress. Int J Cardiol 112:223-228

28. Blair SN, Kampert JB, Kohl HW 3rd, Barlow CE, Macera CA, Paffenbarger RS Jr, Gibbons LW (1996) Influences of cardiorespiratory fitness and other precursors on cardiovascular disease and all-cause mortality in men and women. Jama 276:205-210

29. Blair SN, Kohl HW 3rd, Paffenbarger RS Jr, Clark DG, Cooper KH, Gibbons LW (1989) Physical fitness and allcause mortality. A prospective study of healthy men and women. Jama 262:2395-2401

30. Jackson AS, Beard EF, Wier LT, Ross RM, Stuteville JE, Blair SN (1995) Changes in aerobic power of men, ages 25-70 yr. Med Sci Sports Exerc 27:113-120

31. Jackson AS, Wier LT, Ayers GW, Beard EF, Stuteville JE, Blair SN (1996) Changes in aerobic power of women, ages 20-64 yr. Med Sci Sports Exerc 28:884-891
32. Fleisher LA, Beckman JA, Brown KA, Calkins H, Chaikof E, Fleischmann KE, Freeman WK, Froehlich JB, Kasper EK, Kersten JR, Riegel B, Robb JF, Smith SC Jr, Jacobs AK, Adams CD, Anderson JL, Antman EM, Buller CE, Creager MA, Ettinger SM, Faxon DP, Fuster V, Halperin JL, Hiratzka LF, Hunt SA, Lytle BW, Nishimura R, Ornato JP, Page RL, Tarkington LG, Yancy CW (2007) Acc/aha 2007 guidelines on perioperative cardiovascular evaluation and care for noncardiac surgery: a report of the American college of cardiology/American heart association task force on practice guidelines (writing committee to revise the 2002 guidelines on perioperative cardiovascular evaluation for noncardiac surgery): developed in collaboration with the American society of echocardiography, American society of nuclear cardiology, heart rhythm society, society of cardiovascular anesthesiologists, society for cardiovascular angiography and interventions, society for vascular medicine and biology, and society for vascular surgery. Circulation 116:e418-e499 